\title{
Optimal Model of Forecasting the Per Capita Disposable Income of Urban Residents
}

\author{
Zhongwen Chen \\ Department of Science and Technology, Huanggang Normal University \\ Huanggang 438000, Hubei Province, China \\ zhongwenchen@foxmail.com
}

\begin{abstract}
Keywords:The per capita disposable income; Optimization forecasting model; Power function transformation; Improved GM(1,1) model

Abstract. The per capita disposable income of urban residents reflects the people's living standards and spending power. In this paper, we study the problem of forecasting the per capita disposable income of urban residents, and present an optimization forecasting model based on the grey system theory, i.e., an improved $\operatorname{GM}(1,1)$ model with the power function transformation. Moreover, an application example of forecasting the per capita disposable income is provided to highlight the implementation, practicality, and effectiveness of the presented model.
\end{abstract}

\section{Introduction}

Disposable income of residents is a statistical concept, which is different from resident income. Generally speaking, disposable income of residents is the income which can be used discretionary for the residents. It is subtracted from the total household income in the payment of taxes to the country, after deducting the pay social insurance, such as the remaining income medical insurance, endowment insurance, unemployment insurance and so on. These deductions are paid in accordance with the tax laws and relevant national regulations of the country, which the residents must pay. Because they can no longer pay to cover other areas. But the commercial insurance of residents can not be deducted. Commercial insurance is not prescribed to purchase by the state. It is also deducted from residents' account in terms of our accounts as subsidies granted. Those are the remaining disposable income of residents.

From the disposable income of residents, the per capita disposable income of residents is very important. Because it marks the spot of the spending power of residents. Does his income increase or decreased? how much spending power for a resident or a family, which depend on this index, because it is disposable, and it can be used for consumption, investment, buy stocks, funds for deposits, the faster growth of this index reflects the people's living standards improve faster, and it also reflects his spending power is stronger. Thus it is a very important basis for national decision-making. I look high and low income mainly depends on the index, if the index than other indicators, such as price and compared, if this indicator is higher than the rise in prices, the actual living standards are improving, and if less than the price the rise in the price index is even higher than him then that the standard of living is actually in decline.

In this paper, we study the problem of forecasting the per capita disposable income of urban residents, and present an optimal model of forecasting the per capita disposable income of urban residents by using the grey system theory [1-2]. Concretely, this paper establishes an improved $\operatorname{GM}(1,1)$ model based on the power function transformation [3-5]. The different values of power $\alpha$ are determined to compare the forecasting precision. The results show that the forecasting precision with power function transformation is higher than the traditional $\operatorname{GM}(1,1)$ model. 


\section{The Optimization Forecasting Model}

In this section, we will present an optimal model of forecasting the per capita disposable income of urban residents based on the $\operatorname{GM}(1,1)$ model. As we know, a original series can be used to make the $\operatorname{GM}(1,1)$ modeling only if its class ratio fall into capacitable covering [1-2], i.e., $\sigma(k) \in\left(e^{-2 /(n+1)}, e^{2 /(n+1)}\right)$,

where $n$ are numbers of the elements in the data series. Moreover, in order to get a high precision $\operatorname{GM}(1,1)$ model, the class ratio $\sigma(k)$ is limited in subinterval $(1-\varepsilon, 1+\varepsilon)$, where $\varepsilon$ is a real number which is small enough. Therefore, the data processing principle is that the class ratio of processed data must approachs 1, i.e. the class ratio deviation must be small as much as possible. From this precondition, here we establish an improved $\operatorname{GM}(1,1)$ model with the power function transformation based on the traditional $\operatorname{GM}(1,1)$ model. Concrectly, using the power function $y^{-\beta}$ transformation for data processing to the original series

$$
y^{(0)}=\left(y^{(0)}(1), y^{(0)}(2), \mathrm{L}, y^{(0)}(n)\right), y^{(0)}(k)>1, k=1,2, \mathrm{~L}, n .
$$

The following Theorem 1 solves the problem of determining the value of $\beta$ which should be satisfied in $\mathrm{GM}(1,1)$ modeling.

Theorem 1. Let $w=y(k) / y(k-1)$, for any $0<\varepsilon<1$, if

$$
0<\beta<\min _{k}\left[\frac{2}{(k+1)|\ln w|}, \frac{\ln (1+\varepsilon)}{\ln w}, \frac{\ln (1-\varepsilon)}{\ln w}\right],
$$

where $\frac{\ln (1+\varepsilon)}{\ln w}>0, \frac{\ln (1-\varepsilon)}{\ln w}>0$, which means we can get high precision in $\operatorname{GM}(1,1)$ modeling if $\beta$ satisfies (1).

Proof. In order to get high precision, the transformation series of power function $y^{-\beta}$ should satisfy the prerequisite_ _ it can be used to establish $\mathrm{GM}(1,1)$ model.

A transformation series of power function $y^{-\beta}$ can be used to establish $\operatorname{GM}(1,1)$ model only if its class ratio $\sigma_{y}(k)$ satisfies the following condition

$$
e^{-\frac{2}{n+1}}<\sigma_{y}(k)<e^{\frac{2}{n+1}}
$$

that is

$$
e^{-\frac{2}{n+1}}<\frac{[y(k-1)]^{-\alpha}}{[y(k)]^{-\alpha}}<e^{\frac{2}{n+1}} .
$$

Together with $w=\frac{y(k)}{y(k-1)}$ and $\beta>0$, Thus

$$
e^{-\frac{2}{n+1}}<w^{\beta}<e^{\frac{2}{n+1}},
$$

so we obtain

$$
-2 /(n+1)<\beta \ln v<2 /(n+1),
$$

i.e., $0<\beta<\min _{k} \frac{2}{(n+1)|\ln w|}$.

Morover, $\delta_{y}(k)<\varepsilon$, so we have

$$
\left|1-\frac{[y(k-1)]^{-\beta}}{[y(k)]^{-\beta}}\right|<\varepsilon,
$$

that is,$-\varepsilon<1-w^{\beta}<\varepsilon$, we can obtain

$$
0<\beta<\min _{k}\left[\frac{2}{(k+1)|\ln w|}, \frac{\ln (1+\varepsilon)}{\ln w}, \frac{\ln (1-\varepsilon)}{\ln w}\right],
$$


where $\frac{\ln (1+\varepsilon)}{\ln w}>0, \frac{\ln (1-\varepsilon)}{\ln w}>0$.

From above power function $y^{-\beta}$ transformation, an improved $\operatorname{GM}(1,1)$ model based on the traditional GM(1,1) model is established.The detailed steps are given as follows.

Step 1: Suppose that the original series is $y^{(0)}=\left(y^{(0)}(1), y^{(0)}(2), \mathrm{L}, y^{(0)}(n)\right), y^{(0)}(k)>1$, $k=1,2, \mathrm{~L}, n$. Using above power function $y^{-\beta}$ transformation to process the original series $y^{(0)}$, where the value of $\beta$ is determined by formula (1). After data processing, the original series $y^{(0)}$ becomes a new series as follows.

$$
\left.\left.y^{(1)}=\left(y^{(1)}(1), y^{(1)}(2), \mathrm{L}, y^{(1)}(n)\right)=\left(\left[y^{(0)}(1)\right]^{-\beta}, y^{(0)}(2)\right]^{-\beta}, \mathrm{L}, y^{(0)}(n)\right]^{-\beta}\right) \text {. }
$$

Step 2: Making 1-AGO for $y^{(1)}$, a transformed series $y^{(2)}$ is obtained, then establish $\operatorname{GM}(1,1)$ grey forecasting model (the modeling process is similar with the traditional $\operatorname{GM}(1,1)$ model).

Step 3: Computing the forecasting values of generating operation series $\hat{y}^{(2)}(k)$ and the transformation series $\hat{y}^{(1)}(k)$, where

$$
\hat{y}^{(1)}(k)=\hat{y}^{(2)}(k)-\hat{y}^{(2)}(k-1), k=2,3, \cdots, n \text {. }
$$

Step 4: Making the error analysis for the improved $\operatorname{GM}(1,1)$ forecasting model.

Step 5: Computing the forecasting values of the original series

$$
\hat{y}^{(0)}(k)=\left[\hat{y}^{(1)}(k)\right]^{-\frac{1}{\beta}}, \quad k=2,3, \cdots, n \text {. }
$$

\section{An Application Example}

In this section, we give an application example of forecasting the per capita disposable income to demonstrate the effectiveness and implementation of above presented improved $\operatorname{GM}(1,1)$ forecasting model.

Now we give an original data of the per capita disposable income for one city from the year 1996 to 2003. Now using above improved GM $(1,1)$ model with power function transformation to forecast the per capita disposable income from the year of 2004 to 2006.

First we use the formula (1) to get the value range of $\beta$ in the power function $y^{-\beta}$ transformation is $0<\beta<0.256$. Now we set $\beta=0.1$, the forecasting model of per capita disposable income is as follows.

$$
\begin{aligned}
& \hat{y}^{(0)}(k)=4614.1 e^{0.084 k}, k \geq 1 . \\
& \hat{y}^{(0)}(1)=4838.9 .
\end{aligned}
$$

Further, we compute other case $\beta=1$, which the values of $\beta$ are not in the feasible range $0<\beta<0.256$. The results are all listed in Table 1 .

Table 1. The forecasting results of the per capita disposable income (unit: yuan)

\begin{tabular}{c|c|c|c|c|c}
\hline \multirow{2}{*}{ Year } & \multirow{2}{*}{$\begin{array}{c}\text { Per capita } \\
\text { disposable } \\
\text { income }\end{array}$} & $\begin{array}{c}\text { Forecasting } \\
\text { value }\end{array}$ & $\begin{array}{c}\text { Relative } \\
\text { error }\end{array}$ & $\begin{array}{c}\text { Forecasting } \\
\text { value }\end{array}$ & $\begin{array}{c}\text { Relative } \\
\text { error }\end{array}$ \\
\cline { 3 - 6 } 1996 & 4838.9 & 4838.9 & $0 \%$ & 4838.9 & $0 \%$ \\
1997 & 5160.3 & 5016.9 & $2.7 \%$ & 5050.5 & $2.2 \%$ \\
1998 & 5425.1 & 5453.19 & $-0.5 \%$ & 5494.5 & $1.3 \%$ \\
1999 & 5854.0 & 5928.29 & $-1.2 \%$ & 5917.2 & $1.1 \%$ \\
2000 & 6280.0 & 6444.8 & $-2.6 \%$ & 6451.6 & $2.8 \%$ \\
2001 & 6859.6 & 7006.3 & $-2.1 \%$ & 6993.1 & $2.0 \%$ \\
2002 & 7702.8 & 7616.7 & $1.1 \%$ & 7518.8 & $2.4 \%$ \\
2003 & 8472.2 & 8280.4 & $2.2 \%$ & 8264.5 & $2.6 \%$ \\
$2004^{*}$ & - & 9001.8 & - & - & - \\
$2005^{*}$ & - & 9786.2 & - & - & - \\
$2006^{*}$ & - & 10638.8 & - & - & - \\
\hline
\end{tabular}

(The original data are excerpted from Chinese statistical yearbook of 2004) 
From the results in Table 1, we can see that the forecasting precision of improved $\operatorname{GM}(1,1)$ model with power function $y^{-\beta}$ transformation are very high, which are greater than $97 \%$. And the forecasting precision with $\beta$ in the feasible range is higher than the precision with $\beta$ which is not in the feasible range. From this results, we can conclude that the improved $\operatorname{GM}(1,1)$ model with power function $y^{-\beta}$ transformation presented in this paper can be used to forecast the per capita disposable income effectively.

\section{Conclusions}

Aiming at the decision problem of forecasting the per capita disposable income of urban residents, this paper presents an improved $\operatorname{GM}(1,1)$ model with the power function transformation based on the traditional $\operatorname{GM}(1,1)$ model, and the feasible value range of the power $\beta$ which should be satisfied in high precision $\operatorname{GM}(1,1)$ modeling is given. It is of a great application value for forecasting the per capita disposable income of urban residents.

\section{Acknowledgements}

This work was supported by the 2013 Humanities and Social Science Planning Fund Project of Ministry of Education (No. 13YJA630010), and the Outstanding Young Innovative Group Project of Hubei Province (No.T201415).

\section{References}

[1] J.L. Deng: The Foundation of Grey Theory (Huazhong University of Science and Technology Press, Wuhan 2002).

[2] J.L. Deng: Grey Forecasting and Grey Decision-making (Huazhong University of Science and Technology Press, Wuhan 2002).

[3] X.P. Xiao, Z.M. Song and F. Li: The Foundation and Application of Grey Technology (Science Press, Beijing 2005).

[4] S.F. Liu and J.L. Deng: The Journal of Grey System Vol. 1 (1999), p. 131-138.

[5] W.Z. Dai and J.F. Li: Systems Engineering Theory \& Practice Vol. 11 (2004), p. 63-67.

[6] Y.H. Lin, P.C. Lee and T.P. Chang: Expert Systems with Application Vol. (2009), p. 780-789. 